\title{
Particle filter implementation of the multi-Bernoulli filter for superpositional sensors
}

\author{
Santosh Nannuru and Mark Coates \\ Department of Electrical and Computer Engineering \\ McGill University \\ Montreal, Canada \\ Email: santosh.nannuru@mail.mcgill.ca, mark.coates@mcgill.ca
}

\begin{abstract}
The multi-Bernoulli filter is a promising method for computationally efficient and accurate multi-target tracking. Computationally tractable approximations of the multi-Bernoulli filter equations for superpositional sensors were recently derived. In this paper we present a particle filter implementation of these approximate update filter equations. We describe how the filter could be employed to address the radio-frequency tomographic tracking task and conduct a simulation study to compare performance with the probability hypothesis density (PHD) and cardinalized probability hypothesis density (CPHD) filters.
\end{abstract}

\section{INTRODUCTION}

In multi-target tracking, we strive to detect and track a possibly time-varying number of targets. The changing number of targets makes tracking the full posterior challenging and computationally expensive. The random finite set (RFS) formulation, introduced by Mahler in [1], [2], provides an elegant framework for computationally efficent multi-target tracking. The probability hypothesis density (PHD) filter [1] and cardinalized probability hypothesis density (CPHD) filter [2] are popular first-moment based filters developed using the random finite set framework.

The PHD and CPHD filters track only the first moment of the posterior. In most cases this leads to a need for postprocessing procedures such as clustering to identify which part of the probability hypothesis density corresponds to each target. The multi-Bernoulli filter, proposed by Mahler in [3] and modified by Vo et al. in [4], is an alternative random finite set filter that approximately propagates the full multi-target distribution. This allows us to eliminate much of the postprocessing overhead, leading to a cleaner and more efficient filter implementation.

In this paper, we address the task of multi-target tracking when superpositional sensors [5] are used to make observations. Superpositional sensors make observations that are functions of (potentially) all the targets present. The "superpositional" property requires that targets contribute to sensor measurements in an additive fashion (see Section II for clarification). Examples of superpositional sensors include direction-of-arrival sensors for linear antenna arrays [6], antenna arrays in multi-user detection for wireless communication networks [7], and radio frequency (RF) tomographic tracking systems [8].
Recently, we derived an approximation of the multiBernoulli filter for superpositional sensors [5]. Although the filter update equations were identified in [5], no method of implementing the filter was specified. The main contribution of this paper is to describe a particle filter implementation of the approximate multi-Bernoulli filter for superpositional sensors.

Related work: Auxiliary particle filter implementations of computationally tractable approximations of the PHD and CPHD filters for superpositional sensors were presented in [9]. Particle implementations of the multi-Bernoulli filter for standard sensors (each sensor makes an independent observation that is related to at most one target) have been discussed in [10]-[13].

\section{PROBlem FORMULATION}

Our multi-target tracking task is to the estimate the number of targets present at each time step and their corresponding states. The system state (target positions, velocities, etc.) at time $k$ is captured by the random finite set $X_{k}=$ $\left\{\mathbf{x}_{k, 1}, \ldots \mathbf{x}_{k, n_{k}}\right\}$ where $n_{k} \geq 0$ is number of targets present at time $k$. We assume that the individual target dynamics are specified by a Markovian model of the form $\mathbf{x}_{k+1, i}=$ $f_{k+1 \mid k}\left(\mathbf{x}_{k, i}, \mathbf{u}_{k}\right)$ where $\mathbf{u}_{k}$ is a noise term. The single target state dimension is $n_{\mathbf{x}}$, so $\mathbf{x}_{k, i} \in R^{n_{\mathbf{x}}} \forall i$.

The superpositional sensors provide measurements $\mathbf{z}_{k}=$ $\left[z_{k}^{1} \ldots z_{k}^{n_{z}}\right]$ at time $k$. Denote the collection of measurements up to time $k$ as $Z^{[k]}=\left\{\mathbf{z}_{1}, \ldots, \mathbf{z}_{k}\right\}$. The superpositional sensor model dictates that the likelihood function relating the observation $\mathbf{z}_{k}$ and true state $X_{k}$ is of the form

$$
\begin{aligned}
h_{\mathbf{z}_{k}}\left(X_{k}\right) & =h_{\mathbf{z}_{k}}\left(r\left(X_{k}\right)\right) \\
& =h_{\mathbf{z}_{k}}\left(\sum_{\mathbf{x} \in X_{k}} g(\mathbf{x})\right)
\end{aligned}
$$

where $h_{\mathbf{z}_{k}}$ is a real-valued function and $g$ and $r$ are (potentially non-linear) functions mapping to vectors of reals. When the sensor observation noise is additive Gaussian with zero mean and covariance matrix $\Sigma_{r}$, the likelihood takes the form

$$
h_{\mathbf{z}_{k}}\left(X_{k}\right)=\mathcal{N}_{\Sigma_{r}}\left(\mathbf{z}_{k}-r\left(X_{k}\right)\right) .
$$

In this paper, we will focus on the Gaussian observation noise case. 


\section{Multi-Bernoulli FILTER FOR SUPERPOSITIONAL} SENSORS

In this section we summarize the prediction and approximate update filter equations for the multi-Bernoulli filter, as derived in [5]. The multi-Bernoulli filter is based on a multiBernoulli modelling of the underlying random finite set (RFS). A multi-Bernoulli RFS is a union of finitely many Bernoulli random finite sets, each of which models the probability of existence and state distribution of a single target.

A Bernoulli random finite set is an empty set with probability $1-r$ or is a singleton set with probability $r$ with its element $\mathbf{x}$ distributed according to the probability density $p(\mathbf{x})$. It is completely described by specifying the parameter set $\{r, p(\mathbf{x})\}$. A multi-Bernoulli RFS $\chi$ with $M$ components is a union of $M$ Bernoulli random finite sets, $\chi=\chi_{1} \cup$ $\chi_{2} \cup \cdots \cup \chi_{M}$, where each of the $\chi_{i}$ is a Bernoulli RFS. Let the parameters of the $i^{\text {th }}$ Bernoulli random finite set be given by $\left\{r_{i}, p_{i}(\mathbf{x})\right\}$. The parameter set of the corresponding multi-Bernoulli RFS is denoted by $\left\{r_{i}, p_{i}(\mathbf{x})\right\}_{i=1}^{M}$. The multiBernoulli filter strives to track these parameters.

\section{A. Multi-Bernoulli filter prediction}

The superpositional observation model does not change the multi-Bernoulli prediction equations [3], [10]. Let $\left\{r_{k, i}, p_{k, i}(\mathbf{x})\right\}_{i=1}^{M_{k}}$ be the parameter set of the posterior multiBernoulli density at time $k$. Let the predicted multi-Bernoulli set have $M_{k+1 \mid k}$ elements. For brevity we use the following abbreviated notation for the predicted parameters: $r_{i}=r_{k+1 \mid k, i}$ and $p_{i}(\mathbf{x})=p_{k+1 \mid k, i}(\mathbf{x})$. The predicted multiBernoulli RFS parameters are

$$
\left\{r_{i}, p_{i}(\mathbf{x})\right\}_{i=1}^{M_{k+1 \mid k}}=\left\{r_{i}^{P}, p_{i}^{P}(\mathbf{x})\right\}_{i=1}^{M_{k}} \cup\left\{r_{i}^{B}, p_{i}^{B}(\mathbf{x})\right\}_{i=M_{k}+1}^{M_{k+1 \mid k}}
$$

where $\left\{r_{i}^{P}, p_{i}^{P}(\mathbf{x})\right\}_{i=1}^{M_{k}}$ are the parameters of targets propagated from the previous time step and $\left\{r_{i}^{B}, p_{i}^{B}(\mathbf{x})\right\}_{i=M_{k}+1}^{M_{k+1 \mid k}}$ are the parameters of newly born targets. The predicted target parameters at time $k+1$ are related to the posterior parameters at time $k$ as

$$
r_{i}^{P}=r_{k, i} \times\left\langle p_{k, i}, p_{s}\right\rangle, \quad p_{i}^{P}(\mathbf{x})=\frac{\left\langle f_{k+1 \mid k}(\mathbf{x} \mid \cdot), p_{k, i} p_{s}\right\rangle}{\left\langle p_{k, i}, p_{s}\right\rangle}
$$

where the scalar product is defined as $\langle a, b\rangle=\int a(\mathbf{x}) b(\mathbf{x}) d \mathbf{x}$ and $p_{s}(\mathbf{x})$ is the survival probability.

\section{B. Approximate multi-Bernoulli filter update}

Using the following abbreviated notation for the updated parameters, $r_{i}^{\prime}=r_{k+1 \mid k+1, i}$ and $p_{i}^{\prime}(\mathbf{x})=p_{k+1 \mid k+1, i}^{\prime}(\mathbf{x})$, the following approximate filter update equation was derived in [5] for the case of Gaussian observation noise:

$$
r_{i}^{\prime} \cdot p_{i}^{\prime}(\mathbf{x}) \approx r_{i} \cdot p_{i}(\mathbf{x}) \cdot \frac{\mathcal{N}_{\Sigma_{r}+C_{k+1}^{\bar{i}}}\left(\mathbf{z}_{k+1}-g(\mathbf{x})-\mu_{k+1}^{\bar{i}}\right)}{\mathcal{N}_{\Sigma_{r}+C_{k+1}}\left(\mathbf{z}_{k+1}-\mu_{k+1}\right)}
$$

This expression involves the following statistics:

$$
\begin{aligned}
\mu_{k+1} & =\sum_{i=1}^{M_{k+1 \mid k}} r_{i} \cdot s_{i} \\
C_{k+1} & =\sum_{i=1}^{M_{k+1 \mid k}}\left(r_{i} \cdot v_{i}-r_{i}^{2} \cdot s_{i} s_{i}^{T}\right)
\end{aligned}
$$

where $s_{i}=\left\langle p_{i}, g\right\rangle, v_{i}=\left\langle p_{i}, g g^{T}\right\rangle$ and

$$
\begin{aligned}
& \mu_{k+1}^{\bar{i}}=\mu_{k+1}-r_{i} \cdot s_{i} \\
& C_{k+1}^{\bar{i}}=C_{k+1}-\left(r_{i} \cdot v_{i}-r_{i}^{2} \cdot s_{i} s_{i}^{T}\right) .
\end{aligned}
$$

Please refer to [5] for the derivations and more detail.

\section{Multi-Bernoulli filter cardinality update}

Approximate update equations for the cardinality distribution $\pi_{k}(n)$ were also provided in [5]:

$$
\pi_{k+1}(n) \approx \pi_{k+1 \mid k}(n) \cdot \frac{\mathcal{N}_{\Sigma_{r}+C_{k+1}^{n}}\left(\mathbf{z}_{k+1}-\mu_{k+1}^{n}\right)}{\mathcal{N}_{\Sigma_{r}+C_{k+1}}\left(\mathbf{z}_{k+1}-\mu_{k+1}\right)}
$$

This expression involves the following statistics:

$$
\begin{aligned}
\mu_{k+1}^{n} & =\frac{1}{\pi_{k+1 \mid k}(n)} \sum_{i=1}^{M_{k+1 \mid k}} r_{i} \cdot \pi_{k+1 \mid k}^{\bar{i}}(n-1) \cdot s_{i} \\
C_{k+1}^{n} & =\frac{1}{\pi_{k+1 \mid k}(n)}\left(\sum_{i=1}^{M_{k+1 \mid k}} r_{i} \cdot \pi_{k+1 \mid k}^{\bar{i}}(n-1) \cdot v_{i}\right. \\
& \left.+\sum_{i \neq j} r_{i} \cdot r_{j} \cdot \pi_{k+1 \mid k}^{\bar{i}, \bar{j}}(n-2) \cdot s_{i} s_{j}^{T}\right)-\mu_{k+1}^{n}\left(\mu_{k+1}^{n}\right)^{T}
\end{aligned}
$$

where $\pi_{k+1 \mid k}^{\bar{i}}(\cdot)$ is the cardinality distribution excluding the $i^{t h}$ component of the multi-Bernoulli RFS and $\pi_{k+1 \mid k}^{\bar{i}, \bar{j}}(\cdot)$ excludes both the $i^{\text {th }}$ and $j^{\text {th }}$ components.

\section{PARTiCle implementation OF MULTi-BeRnOUlli FILTER}

Pseudocode for a particle filter based implementation of the multi-Bernoulli filter is given in Algorithm 1. For clarity, we have presented a version where only one new target can appear at each time step, but the generalization is straightforward. The posterior multi-Bernoulli RFS at time step $k$ has $M_{k}$ components with parameters given by $\left\{r_{k, i}, p_{k, i}(\mathbf{x})\right\}_{i=1}^{M_{k}}$. The distribution $p_{k, i}(\mathbf{x})$ for each component is approximated using a weighted set of $N_{p}$ particles

$$
p_{k, i}(\mathbf{x}) \approx \sum_{j=1}^{N_{p}} w_{k, i}^{(j)} \delta_{\mathbf{x}_{k, i}^{(j)}}(\mathbf{x})
$$

The multi-Bernoulli prediction equations are used in the prediction step of the algorithm to update the parameters. The target survival probability is assumed to be a constant $p_{s}$ and birth probability for a new target is $p_{b}$ (lines 4-14). One new target is added per iteration step to account for new born targets. In the absence of a specified prior for the birth distribution, the particles for a new target are chosen uniformly within the observation region. 
The required statistics of the mean and covariance matrix are estimated using the particles (lines 15-21). The approximate update equation (3) is used to update the particle weights. The sum of the updated particle weights provides an estimate of the existence probability. Resampling is performed after the weight update. Multiple methods can be employed to estimate the cardinality. Here we employ a simple thresholding of the existence probability $r_{k, i}$ with threshold parameter $r_{0}$. Only the targets above this threshold are carried forward to the next time step. Location estimates are obtained by a simple averaging of all the particles for each component.

\section{A. Computational complexity}

In this section we examine the computational complexity of the algorithm outlined in the previous section. The prediction step has complexity of the order $\mathcal{O}\left(M N_{p}\right)$. Let the observation dimension be $N_{z}$. Estimating the statistics of $\mu$ and $C$ is order $\mathcal{O}\left(M N_{p} N_{z}+M N_{p} N_{z}^{2}\right)$. The update step requires inverting matrices for each component, each of which takes $\mathcal{O}\left(N_{z}^{3}\right)$. Thus the update step requires computations of the order $\mathcal{O}\left(M N_{p}+M N_{z}^{3}\right)$. The resampling step is $\mathcal{O}\left(M N_{p}\right)$. Thus the overall computational complexity is of the order

$$
\begin{aligned}
\mathcal{O}\left(M N_{p}+M N_{p} N_{z}\right. & \left.+M N_{p} N_{z}^{2}+M N_{p}+M N_{z}^{3}+M N_{p}\right) \\
& \approx \mathcal{O}\left(M N_{p} N_{z}^{2}+M N_{z}^{3}\right)
\end{aligned}
$$

\section{NUMERICAL SIMULATIONS}

In this section we evaluate the multi-Bernoulli (MBR) filter in multi-target tracking application using the radio-frequency (RF) tomography setup [14]. We compare the MBR filter with the auxiliary particle filter implementations of the PHD and CPHD filters [9] for superpositional sensors.

The true target trajectories are shown in Figure 2. A single target state consists of its location in the $x-y$ plane and its velocity. The trajectories are generated using linear Gaussian dynamics (see [9] for more detail) with a sampling period of $T=0.25 \mathrm{~s}$ and noise variance of $0.35 \mathrm{~m}^{2}$.

\section{A. Radio-frequency tomography}

A detailed description of the Radio-frequency tomography setup and the RF tomography multi-target measurement model can be found in [9], [14]. A network of $N$ sensors forming $N_{z}=N(N-1) / 2$ unique bidirectional links is used to survey a region, generating $N_{z}$ measurements each time step. The $j^{\text {th }}$ link measurement $z_{k}^{j}$ at time step $k$ is:

$$
\begin{aligned}
& z_{k}^{j}=r^{j}\left(X_{k}\right)+\mathbf{v}_{k}^{j}=\sum_{\mathbf{x} \in X_{k}} g^{j}(\mathbf{x})+\mathbf{v}_{k}^{j} \\
& \text { where, } g^{j}(\mathbf{x})=\phi \exp \left(-\frac{\lambda_{j}(\mathbf{x})}{\sigma_{\lambda}}\right) .
\end{aligned}
$$

Here $\lambda_{j}(\mathbf{x})$ is an elliptical distance measure between a target located at $\mathbf{x}$ and link $j$ (see [9] for more details); $\phi$ and $\sigma_{\lambda}$ are fixed parameters based on physical properties of the sensors; $\mathbf{v}_{k}^{j}$ is the zero-mean Gaussian sensor noise. The RF tomography measurement equation has a superpositional form as can be seen by comparing equations (11) and (1). In our
1: Initialize $M_{0}$ existence probabilities $\left\{\mathbf{r}_{0, i}\right\}_{i=1}^{M_{0}}$

: Initialize $M_{0}$ particle filters $\left\{w_{0, i}^{(j)}, \mathbf{x}_{0, i}^{(j)}\right\}_{j=1}^{N_{p}}$

for $k=1$ to $T$ do

\section{Prediction}

for $i=1$ to $M_{k-1}$ do

$r_{k \mid k-1, i}=r_{k-1, i} \times p_{s}$

for $j=1$ to $N_{p}$ do

$$
\begin{aligned}
& \mathbf{x}_{k, i}^{(j)} \sim f_{k \mid k-1}\left(\mathbf{x}_{k, i} \mid \mathbf{x}_{k-1, i}^{(j)}\right) \\
& w_{k \mid k-1, i}^{(j)}=w_{k-1, i}^{(j)} \times p_{s}
\end{aligned}
$$

end for

\section{end for}

add new target: $M_{k \mid k-1}=M_{k-1}+1$

$r_{k \mid k-1, M_{k \mid k-1}}=p_{b}$

initialize $\left\{w_{k \mid k-1, M_{k \mid k-1}}^{(j)}, \mathbf{x}_{k, M_{k \mid k-1}}^{(j)}\right\}_{j=1}^{N_{p}}$

\section{Estimate statistics}

for $i=1$ to $M_{k \mid k-1}$ do

$$
\begin{aligned}
& \widehat{\mathbf{s}}_{k, i}=\sum_{j} w_{k \mid k-1, i}^{(j)} g\left(\mathbf{x}_{k, i}^{(j)}\right) \\
& \widehat{\mathbf{v}}_{k, i}=\sum_{j} w_{k \mid k-1, i}^{(j)} g\left(\mathbf{x}_{k, i}^{(j)}\right) g^{T}\left(\mathbf{x}_{k, i}^{(j)}\right)
\end{aligned}
$$

end for

$$
\begin{aligned}
& \widehat{\boldsymbol{\mu}}_{k}=\sum_{i} r_{k \mid k-1, i} \cdot \widehat{\mathbf{s}}_{k, i} \\
& \widehat{\mathbf{C}}_{k}=\sum_{i}\left(r_{k \mid k-1, i} \cdot \widehat{\mathbf{v}}_{k, i}-r_{k \mid k-1, i}^{2} \cdot \widehat{\mathbf{s}}_{k, i} \widehat{\mathbf{s}}_{k, i}^{T}\right) \\
& \begin{array}{r}
\text { Update weights } \\
\text { for } i=1 \text { to } M_{k \mid k-1}
\end{array} \text { do } \\
& \widehat{\boldsymbol{\mu}}_{k}^{i}=\widehat{\boldsymbol{\mu}}_{k}-r_{k \mid k-1, i} \cdot \widehat{\mathbf{s}}_{k, i} \\
& \widehat{\mathbf{C}}_{k}^{\bar{i}}=\widehat{\mathbf{C}}_{k}-\left(r_{k \mid k-1, i} \cdot \widehat{\mathbf{v}}_{k, i}-r_{k \mid k-1, i}^{2} \cdot \widehat{\mathbf{s}}_{k, i} \widehat{\mathbf{s}}_{k, i}^{T}\right) \\
& \text { for } j=1 \text { to } N_{p} \mathbf{d o} \\
& w_{k, i}^{(j)}=r_{k \mid k-1, i} \times w_{k \mid k-1, i}^{(j)} \\
& \quad \times \frac{\mathcal{N}_{\Sigma_{r}+\widehat{\mathbf{C}}_{k}^{\bar{i}}\left(\mathbf{z}_{k}-g\left(\mathbf{x}_{k, i}^{(j)}\right)-\widehat{\boldsymbol{\mu}}_{k}^{i}\right)}}{\mathcal{N}_{\Sigma_{r}+\widehat{\mathbf{C}}_{k}}\left(\mathbf{z}_{k}-\widehat{\boldsymbol{\mu}}_{k}\right)}
\end{aligned}
$$

end for

$r_{k, i}=\min \left(\sum_{j} w_{k, i}^{(j)}, 1\right)$

end for

resample: $\left\{w_{k, i}^{(j)}, \mathbf{x}_{k, i}^{(j)}\right\}_{j=1}^{N_{p}} \rightarrow\left\{\frac{1}{N_{p}}, \mathbf{x}_{k, i}^{(j)}\right\}_{j=1}^{N_{p}}$

\section{Update cardinality}

for $n=0$ to $M_{k \mid k-1}$ do

estimate $\mu_{k}^{n}$ and $C_{k}^{n}$

end for

$$
\pi_{k}(n)=\pi_{k \mid k-1}(n) \cdot \frac{\mathcal{N}_{\Sigma_{r}+C_{k}^{n}}\left(\mathbf{z}_{k}-\mu_{k}^{n}\right)}{\mathcal{N}_{\Sigma_{r}+\widehat{\mathbf{C}}_{k}}\left(\mathbf{z}_{k}-\widehat{\boldsymbol{\mu}}_{k}\right)}
$$

\section{$\underline{\text { State estimation }}$}

$M_{k}=0$

for $i=1$ to $M_{k \mid k-1}$ do

if $r_{k, i}>r_{0}$ then

cardinality: $M_{k}=M_{k}+1$

end if

end for

end for

Figure 1. Multi-Bernoulli filter particle implementation. 


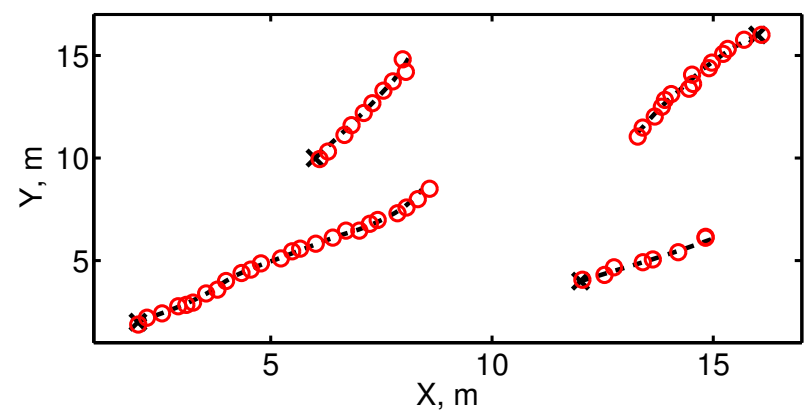

Figure 2. Estimated target location using the multi-Bernoulli filter. Dotted lines show true target trajectories and the circles indicate estimated locations.

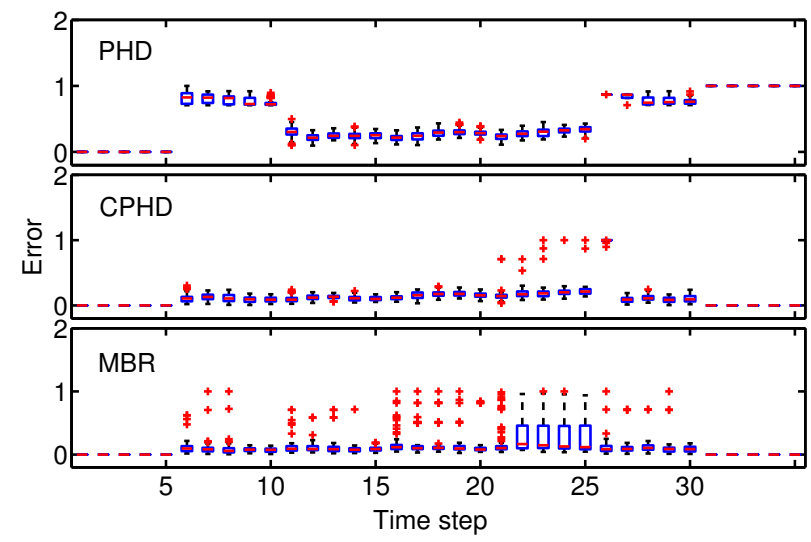

Figure 3. Box-and-whisker plot of OSPA error over time. Boxes indicate 2575 interquartile range; whiskers extend 1.5 times the range and ' + ' symbols indicate outliers lying beyond the whiskers.

simulations we use $N=20$ sensors placed on the periphery of $20 m \times 20 m$ square region. The RF tomography measurement model parameters are $\phi=5$ and $\sigma_{\lambda}=0.4$. The measurement noise variance is $\sigma_{r}^{2}=0.25$.

\section{B. Simulations and results}

We execute multiple (100) Monte Carlo simulations with different random initializations to compare performance. The same target trajectories (as shown in Figure 2) are used in all the simulations. The number of targets present varies from 1 to 4 over the course of the simulation. Table I summarizes the average optimal subpattern assignment (OSPA) error [15] for different algorithms. The error is calculated using three different vales of the cardinality penalty factor $c=1,2.5$ and 5. Cardinality is estimated by thresholding the existence probability $\left(\mathbf{r}_{i}\right)$ using the threshold $r_{0}=0.2$.

\begin{tabular}{|c|c|c|c|}
\hline & \multicolumn{3}{|c|}{ OSPA error } \\
\hline Algorithm & $c=1$ & $c=2.5$ & $c=5$ \\
\hline PHD & 0.49 & 1.03 & 1.95 \\
\hline CPHD & 0.12 & 0.15 & 0.16 \\
\hline MBR & 0.11 & 0.18 & 0.30 \\
\hline
\end{tabular}

Table I

RADIO-FREQUENCY TOMOGRAPHY MULTI-TARGET TRACKING: AVERAGE OSPA ERROR FOR PHD, CPHD AND MBR FILTERS.

The MBR filter has a slightly smaller error than the CPHD filter when the cardinality error penalty is small $(c=1)$ but a larger error as the value of $c$ is increased. This indicates that MBR makes more cardinality errors compared to the CPHD but is slightly more accurate in estimating the target locations. Figure 2 shows example estimated target trajectories.

Figure 3 plots the box-and-whisker plot of the average OSPA error over time combining data over 100 Monte Carlo simulations with cardinality penalty factor $c=1$. The MBR filter has more outliers than CPHD filter due to cardinality errors, but the median error is smaller.

\section{CONCLUSIONS}

We have presented a particle filter implementation of the multi-Bernoulli filter equations for superpositional sensors. We examined the performance of the proposed filter using numerical simulations of radio-frequency tomographic tracking. The multi-Bernoulli filter has more cardinality errors than the CPHD filter but its median location error is otherwise smaller. The filter poses a reduced computational overhead because it avoids the costly clustering step.

\section{REFERENCES}

[1] R. Mahler, "Multitarget Bayes filtering via first-order multitarget moments," IEEE Trans. Aerospace and Electronic Systems, vol. 39, no. 4, pp. 1152-1178, Oct. 2003.

[2] - "PHD filters of higher order in target number," IEEE Trans. Aerospace and Electronic Systems, vol. 43, pp. 1523-1543, Oct. 2007.

[3] — Statistical multisource-multitarget information fusion. Artech House, Boston, 2007.

[4] B. Vo, B. Vo, and A. Cantoni, "The cardinality balanced multi-target multi-Bernoulli filter and its implementations," IEEE Trans. Signal Proc., vol. 57, no. 2, pp. 409-423, 2009.

[5] S. Nannuru and M. Coates, "Multi-Bernoulli filter for superpositional sensors," in Proc. Int. Conf. Inf. Fusion, Istanbul, Turkey, Jul. 2013.

[6] B. Balakumar, A. Sinha, T. Kirubarajan, and J. Reilly, "PHD filtering for tracking an unknown number of sources using an array of sensors," in Proc. Workshop Stat. Sig. Proc., Bordeaux, France, Jul. 2005.

[7] D. Angelosante, E. Biglieri, and M. Lops, "Multiuser detection in a dynamic environment: Joint user identification and parameter estimation," in Proc. IEEE Int. Symp. Inf. Theory, Nice, France, Jun. 2007.

[8] X. Chen, A. Edelstein, Y. Li, M. Coates, M. Rabbat, and A. Men, "Sequential Monte Carlo for simultaneous passive device-free tracking and sensor localization using received signal strength measurements," in Proc. Int. Conf. Inf. Proc. Sens. Networks, Chicago, IL, U.S.A., April 2011.

[9] S. Nannuru, M. Coates, and R. Mahler, "Computationally-tractable approximate PHD and CPHD filters for superpositional sensors," IEEE J. Sel. Topics in Sig. Proc., vol. 7, no. 3, pp. 410-420, 2013.

[10] B. Vo, B. Vo, N. Pham, and D. Suter, "Joint detection and estimation of multiple objects from image observations," IEEE Trans. Signal Proc., vol. 58, no. 10 , pp. 5129-5141, 2010.

[11] R. Hoseinnezhad, B. Vo, and B. Vo, "Visual tracking in background subtracted image sequences via multi-Bernoulli filtering," IEEE Trans. Signal Proc., vol. 61, no. 2, pp. 392-397, 2013.

[12] J. Williams, "Hybrid Poisson and multi-Bernoulli filters," in Proc. Int. Conf. Information Fusion, Singapore, Jul. 2012.

[13] F. Lian, C. Li, C. Han, and H. Chen, "Convergence analysis for the SMC-MeMBer and SMC-CBMeMBer filters," J. of App. Mathematics, vol. 2012, 2012.

[14] F. Thouin, S. Nannuru, and M. Coates, "Multi-target tracking for measurement models with additive contributions," in Proc. Int. Conf. Information Fusion, Chicago, IL, U.S.A., Jul. 2011.

[15] D. Schuhmacher, B.-T. Vo, and B.-N. Vo, "A consistent metric for performance evaluation of multi-object filters," IEEE Trans. Signal Proc., vol. 56, no. 8, pp. 3447-3457, Aug. 2008. 